\title{
An intelligent spectrum handoff scheme based on multiple attribute decision making for LTE-A network
}

\author{
Abdulraqeb Alhammadi ${ }^{1}$, Mardeni Roslee ${ }^{2}$, Mohamad Yusoff Alias ${ }^{3}$, Khalid Sheikhidris ${ }^{4}$ \\ Yong Jun Jack ${ }^{5}$, Anas Bin Abas ${ }^{6}$, Kesh. S. Randhava ${ }^{7}$ \\ ${ }^{1,2,3,4,7}$ Center of Wireless Technology, Faculty of Engineering, Multimedia University, Malaysia \\ ${ }^{5}$ Webe Digital Sdn Bhd, 159, Jalan Templer, Seksyen 8, Petaling Jaya, Malaysia \\ ${ }^{6}$ Telekom Research and Development Sdn Bhd, TM Innovation Centre, Lingkaran Teknokrat Timur, Malaysia
}

\begin{tabular}{|c|c|}
\hline Article Info & ABSTRACT \\
\hline & \multirow{9}{*}{$\begin{array}{l}\text { Cognitive radio networks (CRNs) play an important role in wireless } \\
\text { communications which have the ability to significantly utilize the spectrum } \\
\text { that is not in used and reduce the current spectrum scarcity. CR allows } \\
\text { unlicensed users (secondary users) to occupy the licensed spectrums without } \\
\text { causing interference with licensed users (primary users). This can be } \\
\text { achieved smoothly through four main CR procedures: spectrum sensing, } \\
\text { spectrum decision, spectrum sharing, and spectrum mobility. In this paper, } \\
\text { we propose an intelligent spectrum handoff (HO) scheme based on multiple } \\
\text { attribute decision making using a fuzzy logic controller. The HO decision } \\
\text { depends on three considered parameters: received power, traffic load and } \\
\text { arrival rate of the primary users. The simulation results show the proposed } \\
\text { scheme reduces the unnecessary HOs and the interference which leads to } \\
\text { seamless and efficient communication. }\end{array}$} \\
\hline Received Aug 25, 2018 & \\
\hline Revised Dec 23, 2018 & \\
\hline Accepted Jul 2, 2 & \\
\hline Keywords: & \\
\hline Cognitive radio & \\
\hline Spectrum handoff & \\
\hline Fuzzy logic & \\
\hline LTE-A & \\
\hline
\end{tabular}

Copyright $@ 2019$ Institute of Advanced Engineering and Science. All rights reserved.

\section{Corresponding Author:}

Mardeni Roslee,

Center of Wireless Technology, Faculty of Engineering,

Multimedia University,

63100, Cyberjaya, Selangor, Malaysia.

Email: mardeni.roslee@mmu.edu.my

\section{INTRODUCTION}

Immovable and rigid cellular systems have been failing to cope with the recent growing communication demands due to the lack of predefined resources available for use, as well as the continuously expanding data exchange rates [1]. However, cognitive radio technologies were presented to alleviate the concerns of arbitrary reuse of the spectrum, which may introduce interference [2,3]. Cognitive radio technologies grant users a smooth access to white bands to relieve the licensed spectrum and further prevent interference. Additionally, users are classified as either Primary Users (PUs) who use the existing licensed spectrum, or Secondary Users (SUs) who are obliged to hop on the white band. Accordingly, the US Federal Communication Commission (FCC) limited access of PU to distinct frequencies, and SUs to certain frequencies.

To smoothly access resources, the spectrum sensing process takes place to identify the available accessible bands in the environment. This is considered as the most important stage since it helps to avoid any potential overlapping between PUs and SUs $[4,5]$. These accessible bands will be further evaluated to select and associate the one with the least amount of interference to SUs. However, SUs are obliged to immediately hop off to another vacant band in case any PUs are trying to access the band. Hence, Spectrum Handoff (SHO) must be efficient in identifying vacant bands and taking immediate hopping decisions which require some pre-knowledge about low traffic spectrums [6]. Figure 1 shows the SHO mechanism of transition of spectrum bands for users in Cognitive Radio (CR) networks. It presents that there are three 
spectrum bands which have some vacant channels. The SU occupies the available channel 1 in spectrum band 1 and starts its connections. When the licensed user (PU) arrives to occupy its licensed band, the unlicensed user (SU) must stop its connection and switches to another available channel in other spectrum bands and so on.

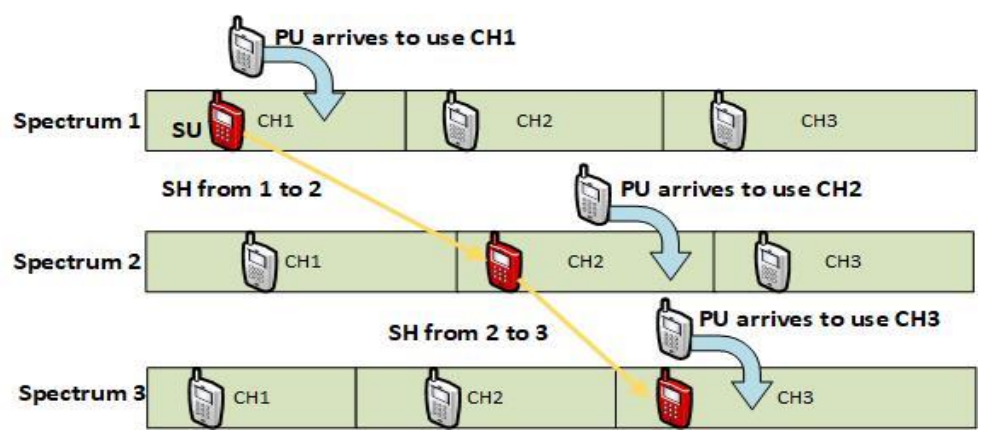

Figure 1. SHO mechanism

The SHO was enhanced to attain adequate and seamless connectivity by various fuzzy logic, intelligence techniques using mathematical tools that can compose real-time decisions. Similarly, the authors of [7] presented an SHO scheme that considers a negotiation approach based on both price and usage-duration. The approach had relatively minimized both numbers of return negotiations and HOs. Nonetheless, non-desired HOs may still occur. Subsequently, the authors of [8,9] proposed a multi-agent negotiation scheme to allow PUs and SUs to share available resources and reduce the number of HOs. Both scheme negotiations with PU depend on minimal prices among the available ones. However, the first scheme negotiates with PUs who hold identical resources, while the second negotiates with few pre-selected PUs. This does not only minimize the HO blocking rate, but also reduces service interruption probabilities. On the other hand, the authors of [10] employed the practical swarm optimization algorithm to accelerate the HO time when a PU requests access to a SUs resource. The scheme reduced the HO delay by approximately 35\%, which also improved the quality of service. Ahmed et al. [11] proposed to foresee the gain of each channel to enhance the spectrum selection process and further improve the Signal-toInterference-plus-Noise Ratio (SINR). The system initially anticipates the gain of all channels and further selects and associates the one with the highest gain prediction. Nevertheless, the availability of multiple channels with different gains may impose some difficulty for easily selecting the perfect channel, which may increase delay itself [12].

The authors of [13] proposed a system that enhances the utilization of the available spectrum to avert incessant switching between channels. Unlike the previous paper, it selects the channel with the least Bit Error Rate (BER) and the highest signal strength. Consequently, the throughput is improved, but PUs' arrivals were not effectively detected. Alternatively, Pham et al. [14] employed the Hidden Markov model to evaluate and oversee the channel status. It arbitrarily selects channels using a proactive SHO algorithm to analyze system efficiency. The authors present a study on signal strength with frequency approaches from $1.8 \mathrm{GHz}$ to $2.2 \mathrm{GHz}$ in indoor environments. It focuses more on mobility of the receiving device, where there will be different distances. In the paper, an USRP (Universal Software Radio Peripheral) device is used as Base Stations (BS) to transmit the desired frequency as well as to receive the signal strength at certain distances in the indoor environment [15]. In another paper, the authors focus on the SH, which manages the movements of SUs to occupy the vacant unlicensed spectrum without interrupting the transmission of PUs [16].

In recent works, there are several research articles have recently been published such as $[7,17,18]$ that discussed the SHO using different algorithms to enhance SHO performance. Although these proposed algorithms provide some enhancement in SHO performance, however, they did not take some important parameters such as traffic load of BS, Reference Signal Received Power (RSRP) and arrival rate of the PUs and SUs into account. These parameters have high impact on the spectrum mobility which allow the SUs to move and utilize the vacant spectrums smoothly. Thus, in this paper, we propose SHO mechanism that utilizes the vacant spectrums using multiple attribute decision making. It also applies the RSRP, traffic readings, and PUs' request rate to effectively administer non-desired HOs. The rest of the paper is organized into four sections. Section II elaborates the system model and the proposed fuzzy logic based on the SH. The performance evaluation of the proposed system and results is presented in Section III. Lastly, Section IV concludes the paper and presents possible future enhancements. 


\section{SYSTEM MODEL AND FUZZY LOGIC SYSTEM}

In this section, we first introduce the system model of the cognitive radio network in Section 2.1, and then Section 2.2 explains the fuzzy logic system. Afterwards, the proposed approach based on fuzzy system is presented in Section 2.3.

\subsection{System model}

We introduce a CRN that is formed by a set of PUs $U_{p}=\left[\begin{array}{llll}U_{p 1} & U_{p 2} & U_{p 3} \ldots U_{p n}\end{array}\right]$ and a set of SUs $U_{s}$ $=\left[\begin{array}{lllll}U_{s 1} & U_{s 2} & U_{s 3} & \ldots & U_{s n}\end{array}\right]$. Users who are located within the overlapping area in between two cells can be categorized as $U_{p} s$ and are associated to the primary network. Conversely, they are categorized as $U_{s}$ if they are associated to the secondary network, as shown in Figure 2. We considered that the primary network bears the licensed spectrum, and three parameters should be used: traffic, RSRP, and the $\mathrm{U}_{\mathrm{p}} \mathrm{s}$ request arrival rate $\left(\lambda_{p}\right)$ in the secondary network. Subsequently, for a $U_{s}$ to hop to a primary network, the condition of Traff $_{\text {ic }}{ }_{P N} \geq \operatorname{Max}$; whereby, $R S R P \leq$ Thshould and $\lambda p<1$ must be fulfilled. $U_{s}$ may also check the RSRP and $\lambda p$ at the desired secondary network before hopping off.

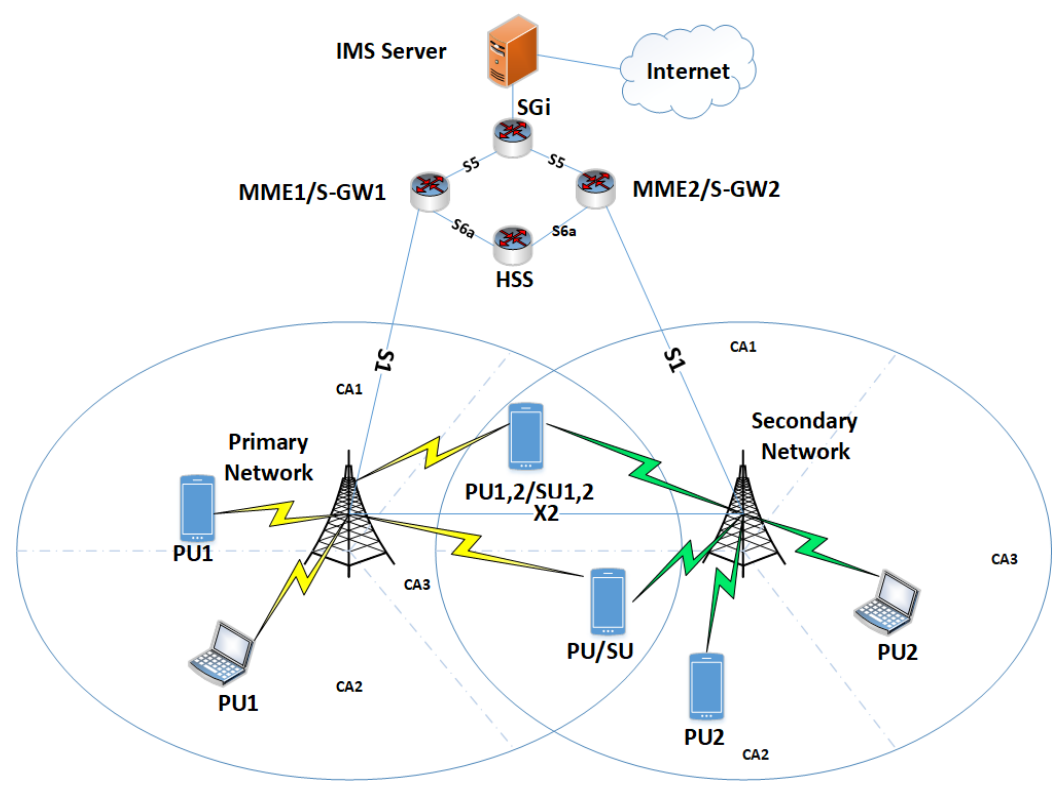

Figure 2. Spectrum hopping in heterogeneous networks

\subsection{Fuzzy logic controller}

One of the recent appealing approaches of spectrum hopping problems in regard to representation and expressions is fuzzy logic. Mathematically, it also deals with relatively high fuzziness-related issues. A Fuzzy Logic Controller (FLC) that consists of elements such as fuzzy interface, fuzzification, and defuzzification as shown in Figure 3 is often needed to apply fuzzy logic concepts

The functionality of the fuzzification block is to receive input parameters (crisp inputs) and logical parameters, respectively. It further processes the input parameters in a similar way to the fuzzy logic of humans. Consequently, the linguistic control rules are used to process the results in a single decision (crisp output) and hence, advanced logical variables are produced; as shown in Figure 4. The input fuzzy variables are represented by input fuzzy sets which consist of a number of membership functions as follows:

$$
\begin{gathered}
\widetilde{X}=\left(x_{1}, x_{2}, \ldots \ldots x_{n}\right) \\
\widetilde{Y}=\left(y_{1}, y_{2}, \ldots \ldots . y_{n}\right) \\
\vdots \\
\widetilde{K}=\left(k_{1}, k_{2}, \ldots \ldots k_{n}\right)
\end{gathered}
$$

where $\tilde{X}, \tilde{Y}$, and $\widetilde{K}$ represent fuzzy sets for input variables $X, Y$, and $K$, respectively. $x_{n} \in \tilde{X} ; y_{n} \in \tilde{Y}$ and $k_{n} \in \widetilde{K}$ are fuzzy membership functions of fuzzy sets $X, Y$, and $K$, respectively, and each has $n$ fuzzy membership functions. 


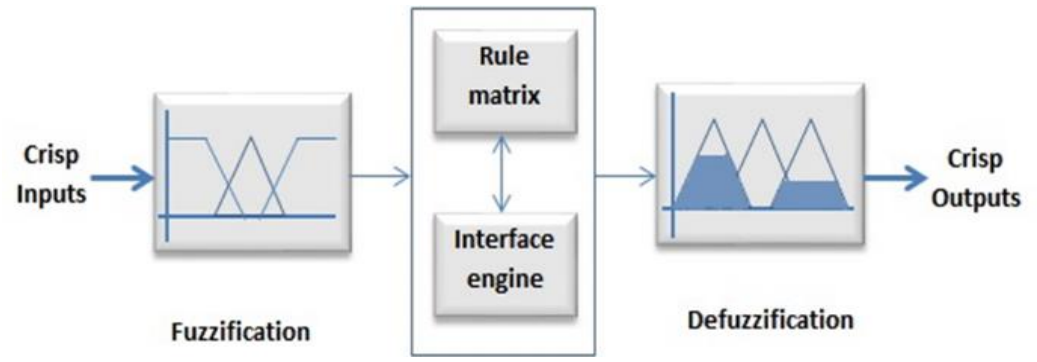

Figure 3. Fuzzy logic controller

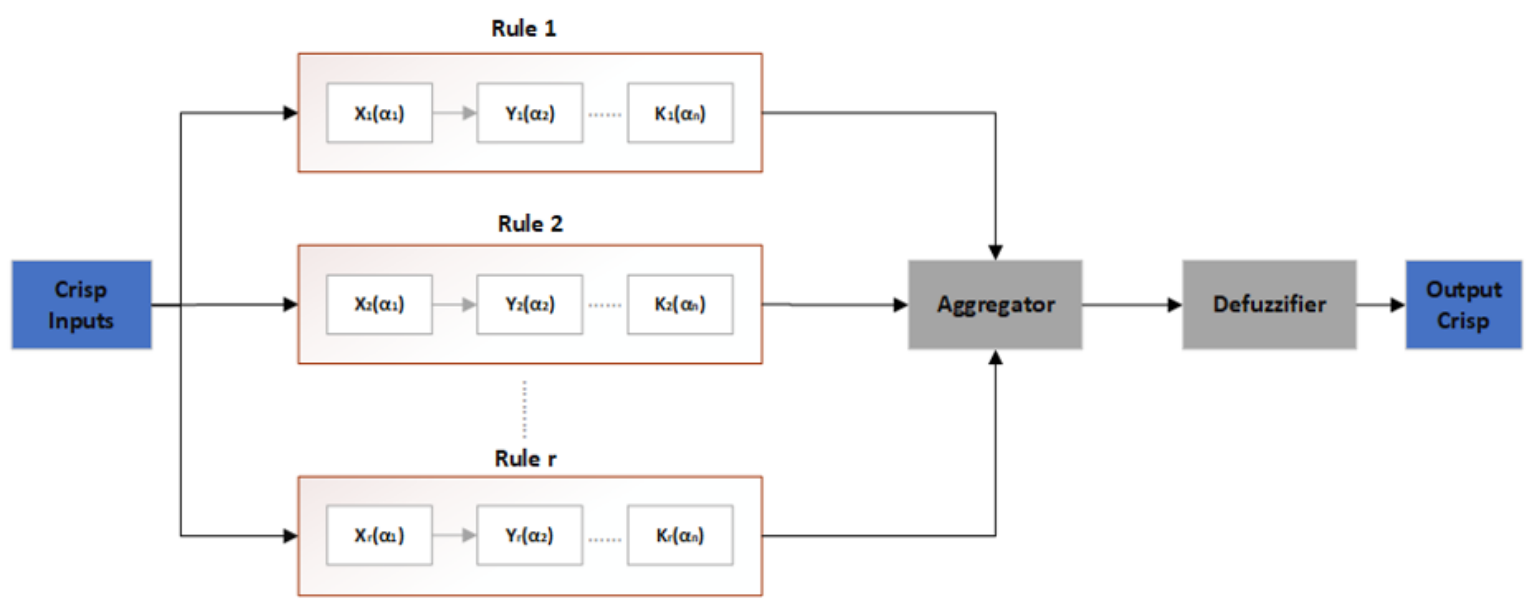

Figure 4. Block diagram of the FLC process

The value of $n$ may or may not be the same in each input fuzzy set. Assuming the same $n$ for all fuzzy sets, the total number of decision rules, $D_{r}$, is calculated using:

$$
D_{r}=n^{s}
$$

where $n$ and $s$ represent the number of fuzzy membership functions in the input fuzzy sets and the total number of fuzzy sets, respectively. Generally, the fuzzy decision rules can be given as follows:

Rule 1: IF $x_{1}$ is true AND $y_{1}$ is false AND ... AND $k_{1}$ is false, THEN $z_{1}$ is true.

Rule 2: IF $x_{2}$ is false AND $y_{2}$ is false AND ... AND $k_{2}$ is false, THEN $z_{2}$ is false.

Rule n: IF $x_{n}$ is false AND $y_{n}$ is tree AND... AND $k_{n}$ is false, THEN $z_{n}$ is true.

where $z_{1}, z_{2}, \ldots \ldots z_{n}$ are the output of fuzzy membership functions defined as:

$$
\tilde{Z}=\left(z_{1}, z_{2}, \ldots \ldots z_{n}\right) .
$$

In this study, an output decision for each individual fuzzy decision rule is obtained using expert knowledge. Although there are several alternative approaches to collect fuzzy decisions via active learning mechanisms and numerical data sets such as colony optimization, neural networks, genetic algorithms, Ad-hoc data covering, etc., their performances are solely restricted to having reliable and precise training sequences which are not always obtainable.

The fuzzy sets, as discussed in [19], and the associated membership functions should primarily be defined in order to determine fuzzified data. Each fuzzy set consists of different levels of variables such as Low, Medium, and High where a level represents a fuzzy membership function. In the fuzzification process, let us say there are many crisp inputs $\alpha_{1}, \alpha_{2}, \ldots, \alpha_{m}$ that are memberships of fuzzified data $\tilde{X}, \widetilde{Y}, \ldots, \widetilde{K}$, respectively. The membership degree of the crisp inputs can be given as follows:

$$
\mu \widetilde{X(}\left(\alpha_{1}\right), \mu \widetilde{Y(}\left(\alpha_{2}\right), \ldots \ldots . \mu \widetilde{K(}\left(\alpha_{m}\right) \in[0.1]
$$


There are two fuzzy inference systems (FIS) which are used in the fuzzification process of fuzzy decisions: Takagi-Sugeno [20] and Mamdani [21]. Nonetheless, the fuzzy decisions in this study were obtained via prior expert knowledge which is a process famously known as fuzzy learning. This study utilized the Mamdani fuzzy inference which enabled us to characterize experts' knowledge more intuitively and in a precise humanized manner. This was due to the unavailability of ready data sets for use.

Formulating the fuzzy control system in Mamdani fuzzy inference with $m$ crisp inputs $\alpha_{1}, \alpha_{2}, \ldots, \alpha_{m}$ and one crisp output $\beta$ is given by:

$$
\text { IF } \alpha_{1} \text { is } \tilde{X}_{i}^{r} \text { and } \alpha_{2} \text { is } \tilde{Y}_{i}^{r} \text { and } \alpha_{m} \text { is } \widetilde{K}_{i}^{r} \text {,THEN } \beta \text { is } \tilde{Z}_{j}^{r}
$$

where $i$ and $j$ are the number of fuzzy membership functions in input and output fuzzy sets, respectively. $r^{\text {th }}$ is the number of rules used for inference, where $r=1,2, \ldots, D_{t}$. The degree of membership function of $\beta$ in the output fuzzy set $\tilde{Z}$ of rule $r^{\text {th }}$ is:

$$
\tilde{Z}^{r}(\beta)=\min \left[\mu \tilde{X}^{r}\left(\alpha_{1}\right), \mu \tilde{Y}^{r}\left(\alpha_{2}\right), \ldots \ldots, \mu \widetilde{K}^{r}\left(\alpha_{n}\right)\right]
$$

The fuzzified data of $\tilde{Z}^{r}(\beta)$ is then aggregated for total number of $D_{t}$ rules which can be given by:

$$
\mu \tilde{Z}(\beta)=\max _{r}\left[\min \left[\mu \tilde{X}^{r}\left(\alpha_{1}\right), \mu \tilde{Y}^{r}\left(\alpha_{2}\right), \ldots, \mu \widetilde{K}^{r}\left(\alpha_{n}\right)\right]\right]
$$

The last process stage is defuzzification which converts the aggregated fuzzified data into a single crisp output with respect to a fuzzy set. There are various defuzzification mechanisms that have already been used in other works. However, in this paper, we considered a centroid defuzzification since it's known to best capture expert knowledge. It can be expressed by:

$$
z^{\dagger}=\frac{\int \mu \tilde{Z}(\beta) \cdot \beta \mathrm{d} \beta}{\int \mu \tilde{Z}(\beta) \mathrm{d} \beta}
$$

\subsection{Proposed HO scheme}

The proposed scheme solely depends on using the FLC, the HO rules, and the three input parameters; as previously highlighted. In spectrum mobility, FIS is applied to make the decision of spectrum access based on the condition of Us parameters such as received power, distance between $U_{p}$ and $U_{s}$, arrival rate of $U_{p}$, and the degree of mobility [22]. In this work, we considered three input parameters that have a significant effect on system performance. These inputs, along with the single output parameter, are shown in Figure 5. FLC quantifies and assesses how possible the $\mathrm{HO}$ is likely to occur based on the input parameters such as the overall traffic, request arrival rate (attained through a Poisson distribution), and the received power. Subsequently, the input parameters that were considered in the proposed scheme are shown in Table 1. Furthermore, Figure 6 presents how the input parameters can contribute to the HO decision.

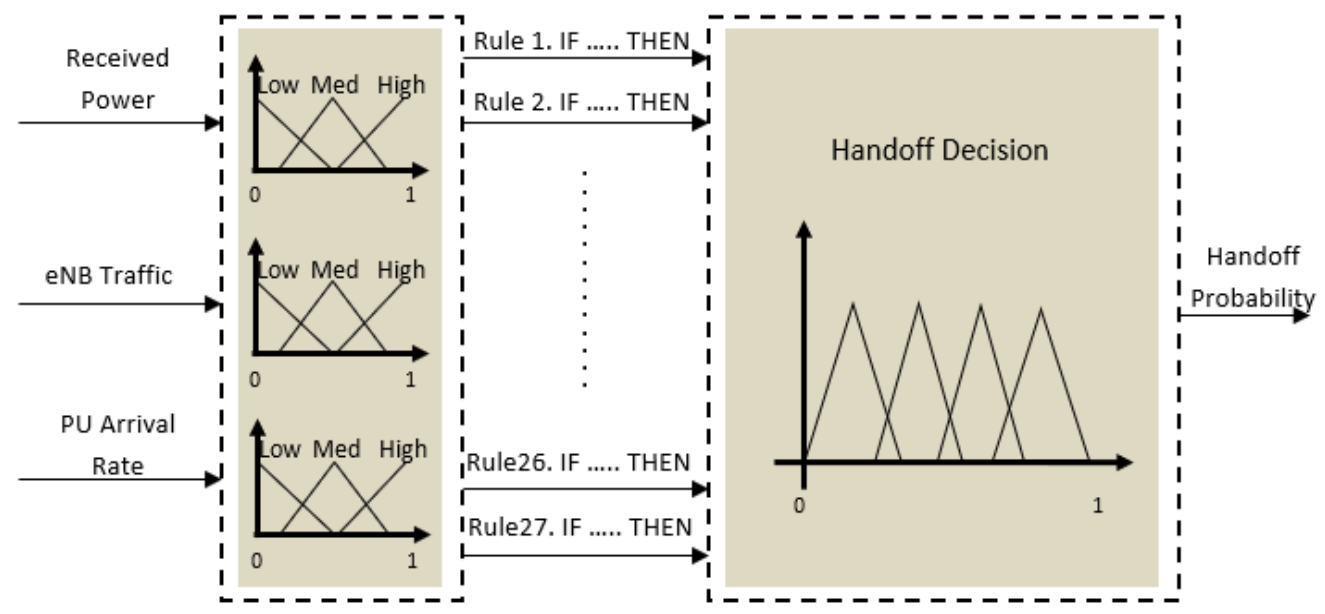

Figure 5. Proposed HO scheme based on FLC 
Table 1. Parameter specification

\begin{tabular}{ll}
\hline Parameters & Values \\
\hline eNB traffic (User) & 10 to 100 \\
PU arrival rate $\lambda \mathrm{p}$ & 0.1 to 1 \\
Received power $(\mathrm{dBm})$ & -115 to -70 \\
\hline
\end{tabular}

Three considered parameters (namely, received power $P_{r}$ at UE, traffic load of eNB $L$, and arrival rate of PUs $\lambda_{\mathrm{p}}$ that have high priority to make a HO decision) are then fed as the input fuzzy set into the fuzzy engine. The three input fuzzy sets for $P_{r}, L$ and $\lambda_{\mathrm{p}}$ are represented by $\widetilde{P_{r}}, \widetilde{L}$, and $\widetilde{\lambda_{p}}$, respectively; whereby, their fuzzy membership functions are defined as ("Low", "Medium", "High").

$$
f\left(P_{r}\right)=f\left(L_{t}\right)=f\left(\lambda_{\mathrm{p}}\right)=\{\text { “' } ", \text { “ } M \text { ", “ } H ”\}
$$

The output fuzzy set is called HO probability which has four fuzzy membership functions ("NO", "Probably No", "Probably Yes", "Yes".).

$$
f(H O)=\{“ Y ”, " P Y ”, " N ”, " P N ”\}
$$

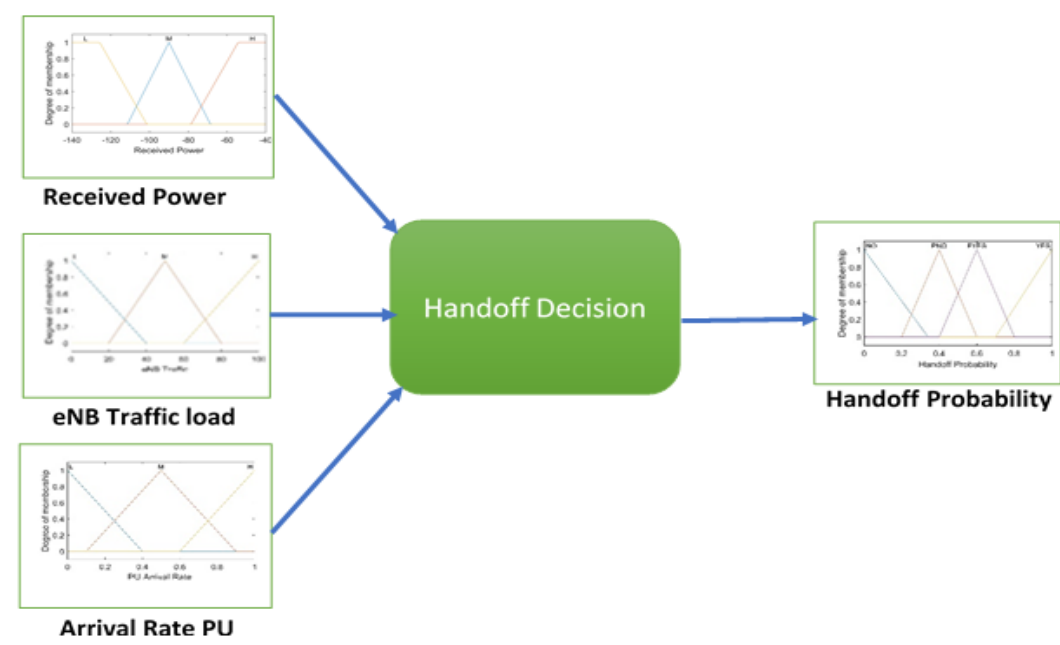

Figure 6. Membership function of the proposed HO scheme

The design of input and output fuzzy sets, a triangular function, is used to develop fuzzy membership functions. Generally, the output decision $\mathrm{HO}$ is set to $N$ (no $\mathrm{HO}$ required) when the highest priority values of $P_{r}$ and $L$ are a member of high and low fuzzy membership functions, respectively. Whereas, $\lambda_{p}$ can be controlled by using spectrum sensing which can be highly considered when $P_{r}$ and $L$ go to low and high, respectively. If the input values of $P_{r}$ and $L_{t}$ are in the medium range, the output decision $H O$ is set to either $P N$ or $P Y$ depending on the input value $\lambda_{p}$. However, once the $P_{r}$ drops to low and $L$ goes to high, the decision output $H O$ is set to $Y$ where $\lambda_{p}$ value ranges between low to medium.

The sequence of fuzzy decision rules required to determine the output decision can be obtained using (1). In this work, the number of fuzzy membership functions in the input fuzzy sets $\mathrm{n}$ are 3 sets, whereas the total number of fuzzy sets s are 3. Therefore, the total number of fuzzy decision rules $D_{t}=n^{s}=$ $3^{3}=27$ rules. Table 2 presents fuzzy decision rules where each rule is assigned to a decision output derived from expert knowledge.

In the next stage, the input parameter values are then fuzzified to generate aggregated fuzzified data using. Mamdani fuzzy inference. The aggregated fuzzified data can be obtained by applying (5) which is given by:

$$
\mu \widetilde{H O}(H O)=\max _{r}\left[\min \left[\begin{array}{c}
\mu \widetilde{P}_{r}^{r}(\text { received power }), \mu{\widetilde{L_{t}}}^{r} \\
(\text { Traffic Load }), \mu \widetilde{\lambda \mathrm{p}^{r}}(\text { arrival rate })
\end{array}\right]\right]
$$


Defuzzifier converts $\mu \widetilde{H O}$ into a crisp value, $\mathrm{HO}^{\dagger}$ which is calculated using (6) as follows:

$$
H O^{\dagger}=\frac{\int \mu \widetilde{H O}(H O) \cdot H O d(H O)}{\int \mu \widetilde{H O}(H O) d(H O)}
$$

where $\mathrm{HO}^{\dagger}$ denotes the defuzzified data generated by the $\mathrm{HO}$ fuzzy engine

\begin{tabular}{|c|c|c|c|c|}
\hline No & $P_{r}$ & $L$ & $\lambda_{p}$ & $\mathrm{P}(\mathrm{HO})$ \\
\hline 1 & Low & Low & Low & $\mathrm{PN}$ \\
\hline 2 & Low & Low & Medium & PY \\
\hline 3 & Low & Low & High & $\mathrm{N}$ \\
\hline 4 & Low & Medium & Low & PY \\
\hline 5 & Low & Medium & Medium & PY \\
\hline 6 & Low & Medium & High & PN \\
\hline 7 & Low & High & Low & $\mathrm{Y}$ \\
\hline 8 & Low & High & Medium & $\mathrm{Y}$ \\
\hline 9 & Low & High & High & PY \\
\hline 10 & Medium & Low & low & $\mathrm{N}$ \\
\hline 11 & Medium & Low & Medium & $\mathrm{N}$ \\
\hline 12 & Medium & Low & High & $\mathrm{N}$ \\
\hline 13 & Medium & Medium & Low & $\mathrm{N}$ \\
\hline 14 & Medium & Medium & Medium & $\mathrm{N}$ \\
\hline 15 & Medium & Medium & High & $\mathrm{N}$ \\
\hline 16 & Medium & High & Low & $\mathrm{Y}$ \\
\hline 17 & Medium & High & Medium & $\mathrm{Y}$ \\
\hline 18 & Medium & High & High & PN \\
\hline 19 & High & Low & Low & $\mathrm{N}$ \\
\hline 20 & High & Low & Medium & $\mathrm{N}$ \\
\hline 21 & High & Low & High & $\mathrm{N}$ \\
\hline 22 & High & Medium & Low & $\mathrm{N}$ \\
\hline 23 & High & Medium & Medium & $\mathrm{N}$ \\
\hline 24 & High & Medium & High & $\mathrm{N}$ \\
\hline 25 & High & High & Low & $\mathrm{Y}$ \\
\hline 26 & High & High & Medium & PY \\
\hline 27 & High & High & High & PN \\
\hline
\end{tabular}

\section{PERFORMANCE EVALUATION}

In this section, the performance evaluation is divided into subsections. Section 3.1 presents the analysis of the input parameters and its impact on the HO probability. Section 3.2 investigates the impact of the proposed HO scheme with different values of consider parameters

\subsection{Analysis of the input parameters}

The performance of the proposed scheme is evaluated through simulations carried out using the MATLAB tool where the values of the input parameters in Table 1 along with the rules in Table 2 are considered. It can be seen in Figure 7 (a) that the HO is much likely to occur when there is relatively high traffic and arrival rate. Consequently, the scheme changes the occupied channel to establish a new transmission on another idle channel.

On the other hand, $\mathrm{HO}$ is likely to occur if request arrival rate increases while the received power degrades below $-115 \mathrm{dBm}$, as presented in Figure 7 (b). Consequently, the scheme will switch the transmission to another idle channel. However, if the received power remains high, the HO decision will solely be built on the request arrival rates.

Additionally, Figure 7 (c) shows the HO decision evaluation based on traffic and received power rates. This shows that the scheme jumps to a certain channel only if it bears less traffic and high received power simultaneously. For this decision to be taken, the two parameter values at the desired station must be satisfied at the connection time.

Figure 8 demonstrates the rules for input and output sets of the fuzzy inference system. It used to monitor the entire implication process from beginning to end. The first three columns indicate the input sets whilst the fourth one indicates to the output set. To test the output set "HO probability" for all rules, the red line adjusts for each observation input set. Setting each input set at different levels yields a ranking of output points, where higher values of the output set indicate a high level of the input sets and vice versa. 


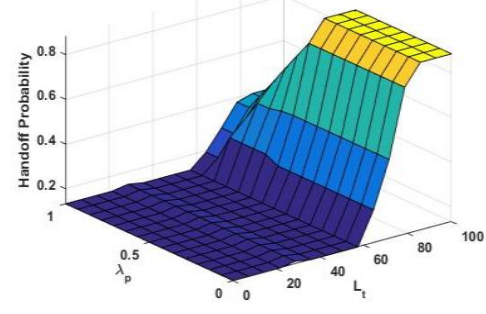

(a)

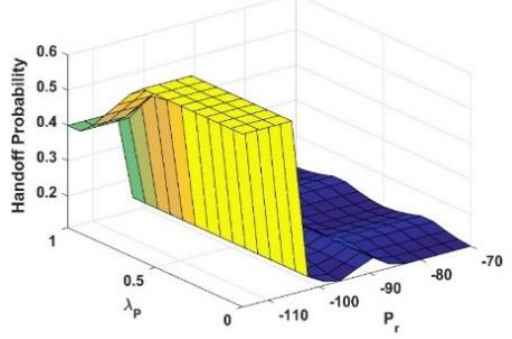

(b)

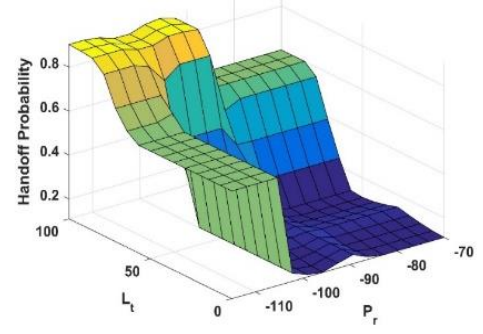

(c)

Figure 7. Probability of $\mathrm{HO}$ with respects to (a) $\lambda_{p}$ and $L_{t}$ (b) $\lambda_{p}$ and $P_{r}$ (c) $P_{r}$ and $L_{t}$

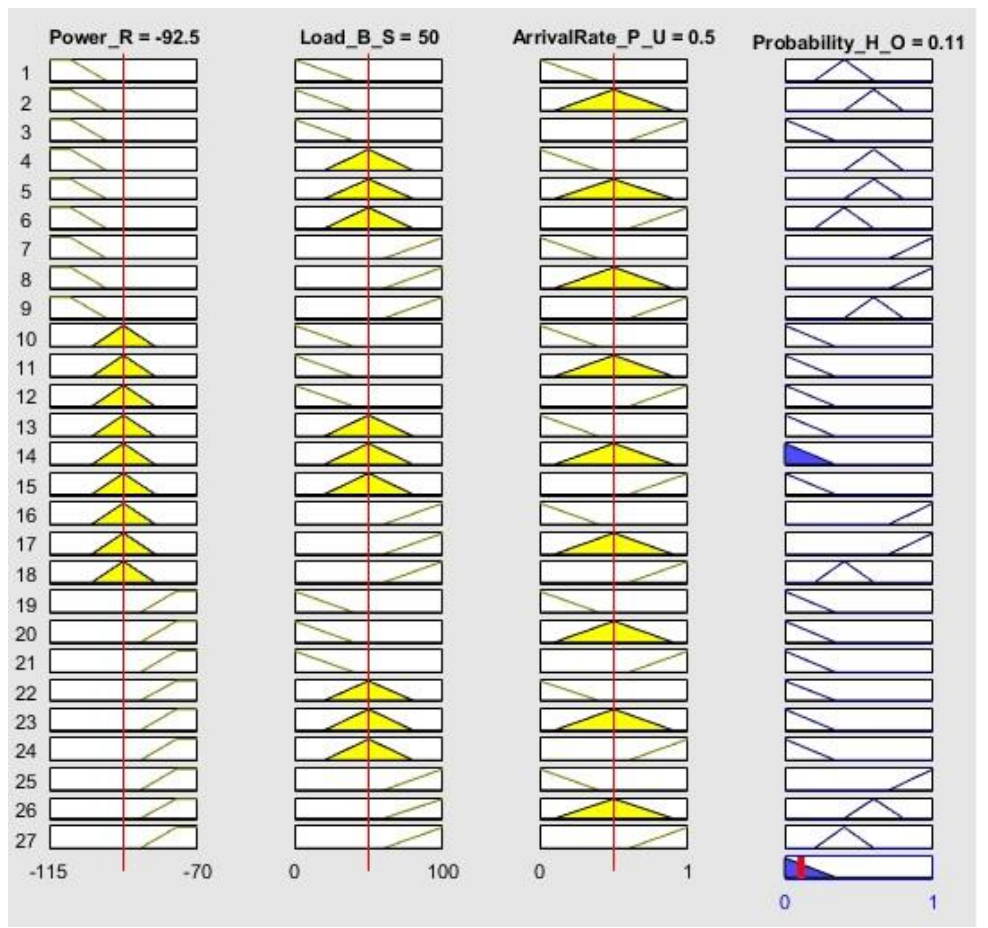

Figure 8. Rule viewer of input and output fuzzy sets

\subsection{Impact of $\mathrm{HO}$ scheme}

The proposed HO scheme was investigated by different rate of $\lambda$ p. Figure 9 and Table 3 present the request arrival rate at three different times $(\lambda \mathrm{p}=0.2,0.5$, and 0.8$)$ with different number of users. It also demonstrates that the $\mathrm{HO}$ only increases as users associated with the BS further increase. Hence, the HO should not be carried to a BS that has high arrival rates or high load. In other words, it is observed that in the case of BS low traffic of arrival rate of PU, the proposed scheme guides the Sus to occupy that spectrum to setup the connection. This will reduce the frequent $\mathrm{HO}$ and searching for other available spectrum bands to continue their connections. On the contrary, high rate of PU arrival and number of users increases the HO probability which affects the network performance.

Figure 10 demonstrates the impact of $P_{r}$ and $L$ on the HO probability. Four different scenarios of $P_{r}$ and $L$ were evaluated with rage of $\lambda_{\mathrm{p}}=0.2$ to 1 with step of 0.2 . It shows that the higher HO probability occurs when the UE communication link becomes very poor and the BS is congested (scenarios 2). However, the HO probability gradually reduces when the $\lambda_{\mathrm{p}}$ of target BS increases. This due to the SUs avoid the interference that might be happening when the higher rate of the PUs arrive to occupy their licensed spectrum. On the other hand, the lower HO probability occurs when the UE communication link becomes very strong and the BS is not fully utilized (scenarios 3). In this case, the HO does not require or becomes unnecessary since $P_{r}$ and $L$ are still in an acceptable level of communication. Thus, reducing of unwanted HOs probability leads to increase the efficiency of communications and reduce the interference. 


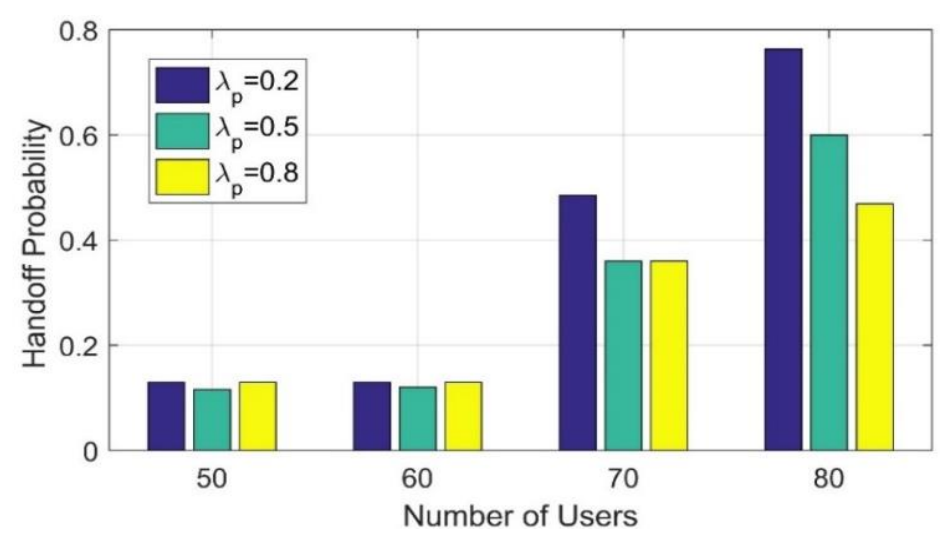

Figure 9. HO probability with different rate of $\lambda_{\mathrm{p}}$

Table 3. HO probability of the proposed scheme with different rate of $\lambda_{\mathrm{p}}$

\begin{tabular}{ccc}
\hline No. of Users & $\lambda_{p}$ & HO Probability \\
\hline \multirow{2}{*}{50} & 0.2 & 0.130 \\
& 0.5 & 0.116 \\
\multirow{2}{*}{60} & 0.8 & 0.130 \\
& 0.2 & 0.131 \\
& 0.5 & 0120 \\
70 & 0.8 & 0.129 \\
& 0.2 & 0.485 \\
& 0.5 & 0.361 \\
\multirow{2}{*}{80} & 0.8 & 0.360 \\
& 0.2 & 0.763 \\
& 0.5 & 0.600 \\
\hline
\end{tabular}

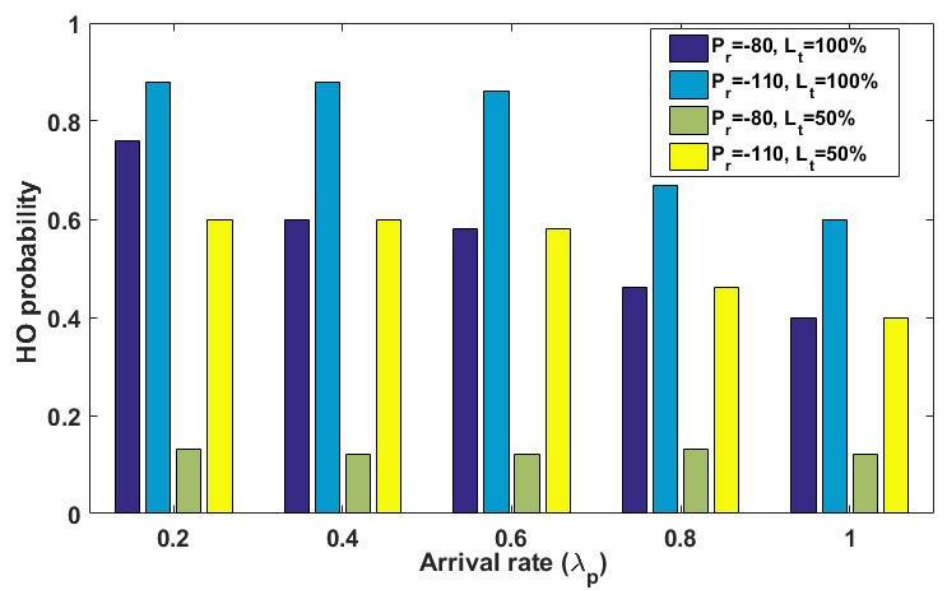

Figure 10. Impact of $P_{r}$ and $L_{t}$ on the HO probability

\section{CONCLUSION}

In this paper, an efficient SHO scheme was proposed based on the fuzzy logic system to avoid the high probability number of HOs. It can be concluded that the proposed fuzzy logic-based algorithm with realistic considerations of traffic, requests arrival rates, and received power leads to relatively longer $\mathrm{HO}$ delays; this subsequently leads to the complete avoidance of unnecessary HOs. Furthermore, reducing of unnecessary HOs leads to reduce the interference occurs when Sus HO to PU licensed spectrum during high arrival rate. This will further stabilize communication and prevent sudden interruptions. Future directions of this topic should apply some intelligence techniques, such as swarm optimizations, to additionally decrease HOs. 


\section{ACKNOWLEDGEMENTS}

This research was supported in part by Telecom Malaysia Research and Development Grant (MMUE170014), Telecom Malaysia.

\section{REFERENCES}

[1] H. Takagi and B. H. Walke, "Spectrum Requirement Planning in Wireless Communications: Model and Methodology for IMT-Advanced", John Wiley \& Sons, Chichester, England, 2008.

[2] S., Khalid, A. M. Yusoff, and R. Mardeni, "Adaptive power management with fractional frequency reuse scheme for co-tier Femto-cell interference reduction," In Communications (MICC), 2017 IEEE 13th Malaysia International Conference on, pp. 311-315. IEEE, 2017.

[3] N. L. Ismail , M. Kassim, M. Ismail and R. Mohamad. "A Review of Low Power Wide Area Technology in Licensed and Unlicensed Spectrum for IoT Use Cases" Bulletin of Electrical Engineering and Informatics, Vol. 7, No. 2, pp. 183 190, 2018.

[4] A. Bhowmick, B. Prasad, S.D. Roy, et al. "Performance of Cognitive Radio Network with Novel Hybrid Spectrum Access Schemes," Wireless Pers Commun, Vol 91, Issue 2, pp 541-560, 2016.

[5] A.S. Khobragade and R.D. Raut, "Hybrid Spectrum Sensing Method for Cognitive Radio," International Journal of Electrical and Computer Engineering (IJECE), Vol.7, No.5, pp. 2683-2695, 2017.

[6] L. De Nardis and M-D-P Guirao, "Mobility aware design of cognitive radio networks: challenges and opportunities", Cognitive radio Oriented Wireless Network and Communication, pp. 1-5, 2010

[7] A. Abdulraqeb, R. Mardeni and Y.A. Mohamad, "Fuzzy Logic Based Negotiation Approach for Spectrum Handoff in Cognitive Radio Network" 2016 IEEE 3rd International Symposium on Telecommunication Technologies (ISTT), Kuala Lumpur, Malaysia, 28 - 30 November 2016.

[8] E. Trigui, M. Esseghir, L. Merghem Boulahia, "Multi-agent systems Negotiation approach for handoff in mobile cognitive radio networkds", IFIP/IEEE NTMS, pp.1-5, 2012.

[9] E Trigui,.; M. Esseghir,.; Boulahia, L.M., "Spectrum handoff algorithm for mobile cognitive radio users based on agents' negotiation," in Wireless and Mobile Computing, Networking and Communications (WiMob), 2013 IEEE 9th International Conference on, vol., no., pp.750-756, 7-9 Oct. 2013.

[10] A. Abdulraqeb, R. Mardeni and Y.A. Mohamad, "Analysis of Spectrum Handoff Schemes in Cognitive Radio Network Using Particle Swarm Optimization” 2016 IEEE 3rd International Symposium on Telecommunication Technologies (ISTT), Kuala Lumpur, Malaysia, 2016.

[11] E. Ahmed, Liu Jie Yao; M. Shiraz, A. Gani,; S. Ali, , "Fuzzy-based spectrum handoff and Channel selection for Cognitive Radio Networks, " in Computer, Control, Informatics and Its Applications (IC3INA), 2013 International Conference on , vol., no., pp.23-28, 19-21 Nov. 2013.

[12] W. Arif, S. Hoque, D. Sen, et al. "A Comprehensive Analysis of Spectrum Handoff Under Different Distribution Models for Cognitive Radio Networks," Wireless Pers Commun, Vol 85, Issue 4, pp 2519-2548, 2015.

[13] P. Maheshwari, A. K Singh, "A fuzzy logic-based approach to spectrum assignment in cognitive radio networks," in Advance Computing Conference (IACC), 2015 IEEE International , vol., no., pp.278-281, 12-13 June 2015.

[14] C. Pham, N.H. Tran, C.T. Do, S. Il Moon and C.S. Hong, "Spectrum handoff model based on hidden markov model in cognitive radio networks", Proc. of IEEE International Conference on Information Networking(ICOIN), pp. 406-441, 2014.

[15] R, Mardeni, A. Abdulraqeb, A. Mohamad Yusoff, A. Khairil, and P. U. Nmenme, "Analysis of LTE-A Signal Strength in Indoor Mobility Environment," Advanced Science Letters Journal, Vol.23, No.11, pp. 11355-11359, ISSN: 1936-6612, 1936-7317.

[16] R, Mardeni, A. Abdulraqeb, A. Mohamad Yusoff, A. Khairil, and P. U. Nmenme. "Efficient handoff spectrum scheme using fuzzy decision making in cognitive radio system." In 2017 rd International Conference on Frontiers of Signal Processing (ICFSP), pp. 72-75. IEEE, 2017.

[17] K. Kumar, A. Prakash and R. Tripathi, "Spectrum handoff scheme with multiple attributes decision making for optimal network selection in cognitive radio networks," Digital Communications and Networks, V. 3, Issue 3, pp 164-175, 2017.

[18] N. Gupta, S. K. Dhurandher, I. Woungang and M. S. Obaidat, "Proactive Decision Based Handoff Scheme for Cognitive Radio Networks," 2018 IEEE International Conference on Communications (ICC), Kansas City, MO, pp. 1-6, 2018.

[19] L. A. Zadeh, "Information and control. Fuzzy Logic," 8(3), 338-353, 1965.

[20] T. Takagi, \& M. Sugeno, "Fuzzy identification of systems and its applications to modeling and control," IEEE Transactions on Systems Man and Cybernetics, 15(1), 116-132. 1985.

[21] E. Mamdani, \& S. Assilian, . "An experiment in linguistic synthesis with a fuzzy logic controller," International Journal of Man-Machine Studies, 7(1), 1-13,1975.

[22] M. Subhedar, \& G. Birajdar. "Comparison of Mamdani and Sugeno Inference Systems for Dynamic Spectrum Allocation in Cognitive Radio Networks," Wireless Pers Communication, Vol 71, Issue 2, pp 805-819, 2013. 